\title{
INJECTIVE MODULES OVER PRUFER RINGS
}

\author{
EBEN MATLIS
}

The purpose of this paper is to find out what can be learned about valuation rings, and more generally Prufer rings, from a study of their injective modules. The concept of an almost maximal valuation ring can be reformulated as a valuation ring such that the images of its quotient field are injective. The integral domains with this latter property are found to be the Prufer rings with a (possibly) weakened form of linear precompactness for their quotient fields. The Prufer rings with linearly compact quotient fields are found to be exactly the maximal valuation rings, and may be characterized as those integral domains $R$ with quotient field $Q$ such that the images of $Q$ are injective and $\operatorname{Hom}_{R}(Q / R, Q / R) \cong R$; or, alternatively, as those integral domains for which a torsion-free submodule $S$ of rank one of a module $B$ is a direct summand whenever $B / S$ is also torsion-free. We are able to rederive many of the results of [2] and [4] by homological methods. Finally, among Noetherian integral domains we characterize the Dedekind rings as those for which every finitely generated torsion module is a direct sum of cyclic modules.

Notation and Definitions. Any ring considered will be commutative with an identity element which acts as the identity operator on any module over the ring. If $A$ is any module, we will denote by $E(A)$ the injective envelope of $A$ [6]. A module $A$ is said to be indecomposable, if it has no proper direct summands. An ideal $I$ of a ring is said to be irreducible, if it is not an intersection of two properly larger ideals. $A$ module over an integral domain will be said to be divisible, if multiplication by any non-zero element of the ring is an epimorphism of the module onto itself; and a module will be said to be torsion-free, if any such multiplication is a monomorphism of the module into itself.

A Prufer ring is an integral domain in which every finitely generated ideal is invertible. A valuation ring is an integral domain in which every two ele-

Received October 7, 1958. 
ments have a greatest common divisor which is equal to one of them. $A$ valuation ring is a local ring in the sense that it has a single maximal ideal. It is easily seen that a ring is a valuation ring if and only if it is a local, Prufer ring. It is also readily verified that an integral domain $R$ with quotient field $Q$ is a valuation ring if and only if the lattice of $R$-submodules of $Q$ (or of $R$ ) is simply ordered. If $R$ is a valuation ring and $S$ a proper submodule of $Q$, then there exists an element $a \neq 0$ in $R$ such that $a \mathrm{~S}$ is an ideal of $R$.

Proposition 1. Let $R$ be a valuation ring with quotient field $Q$. Then

1) $E(R / I)$ is an indecomposable, injective $R$-module for every ideal $I$ of $R$; and every indecomposable, injective $R$-module is of this form.

2) If $I$ is a proper ideal of $R$, then $E(R / I) \cong E(Q / I)$.

3) If $I$ and $J$ are proper ideals of $R$, then $E(R / I) \cong E(R / J)$, if and only if $I \cong J$.

Proof. 1) Since the ideals of $R$ are simply ordered, they are irreducible; and the result follows from [6, Thm. 2.4].

2) As in 1) $E(Q / I)$ is an indecomposable, injective $R$-module. Since $R / I$ is a non-zero submodule of $E(Q / I)$, it follows from [6, Prop. 2.2] that $E(R / I)$ $\cong E(Q / I)$.

3) If $I \cong J$, then there exists $q \neq 0$ in $Q$ such that $I=q J$. Hence $Q / I \cong Q / J$, and by 2) $E(R / I) \cong E(R / J)$. Conversely, if $E(R / I) \cong E(R / J)$, there exists submodules $A$ and $B$ of $E(R / I)$ such that $A \cong R / I$ and $B \cong R / J$. Since $E(R / I)$ is an indecomposable, injective $R$-module, $A \cap B \neq 0$ [6, Prop. 2.2]. If $x \neq 0$ $\in A \cap B$, then the order ideal of $x$ is isomorphic to $I$ and to $J$. Thus $I$ and $J$ are isomorphic.

It follows readily from Proposition 1 that if $S$ and $T$ are $R$-submodules of $Q$, then $Q / S \cong Q / T$, if and only if $S \cong T$. It is also an easy corollary of Proposition 1 that if $A$ is any injective $R$-module, then any element of $A$ is contained in an indecomposable, injective direct summand of $A$. Thus if $B$ is a finitely generated $R$-module, then $E(B)$ is a finite direct sum of indecomposable, injective $R$-modules.

Definition. Let $A$ be a module over a commutative ring $R . A$ is said to be linearly compact if every finitely solvable set of congruences:

$$
x \equiv x_{\alpha} \quad\left(\bmod A_{\alpha}\right)
$$


(where $x_{\alpha} \in A$, and the $A_{\alpha}$ 's are submodules of $A$ ) has a simultaneous solution in $A$. (See [2] and [8]).

We say that $A$ is semi-compact, if the above congruence condition holds whenever the submodules $A_{\alpha}$ are annihilators of ideals of $R$.

$A$ is said to be linearly pre-compact, if every proper homomorphic image of $A$ is linearly compact.

$A$ valuation ring $R$ with quotient field $Q$ is said to be maximal (almost maximal), if $Q$ is linearly compact (linearly precompact). It is easily seen that a valuation ring $R$ is maximal (almost maximal), if and only if $R$ itself is linearly compact (linearly pre-compact) as an $R$-module.

Proposition 2. Let $C$ be an injective module over a commutative ring $R$. Then $C$ is semi-compact.

Proof. Let $x \equiv x_{x}\left(\bmod C_{\alpha}\right)$ be a finitely solvable set of congruences, where $x_{\alpha} \in C$ and $C_{\alpha}$ is the annihilator in $C$ of an ideal $I_{\alpha}$ of $R$. Let $I$ be the ideal of $R$ generated by all of the $I_{\alpha}$ 's. If $a \in I$, then $a \in I_{x_{1}}+\cdots+I_{x_{k}}$. Let $y \in C$ be a solution of the $n$ congruences for the indices $\alpha_{1}, \ldots, \alpha_{n}$. We define an $R$-homomorphism $f: I \rightarrow C$ by $f(a)=a y$. It is easily verified that $f$ is a welldefined homomorphism. Since $C$ is injective, there exists $z \in C$ such that $f(a)$ $=a z$ for every $a \in I$. Then $z$ is a solution of the congruences.

It follows from Proposition 2 that if $C$ is an injective $R$-module such that every submodule of $C$ is the annihilator of some ideal of $R$, then $C$ is linearly compact. In particular, if $R$ is a commutative, complete, Noetherian, local ring, with maximal ideal $M$, then $E(R / M)$ is linearly compact [6, Thm. 4.2].

The following proposition is due to I. Fleischer [2, Lemma 1]; but we include a proof for the sake of completeness.

Proposition 3. A divisible, semi-compact module $D$ over a Prufer ring $R$ is injective.

Proof. Let $I$ be an ideal of $R$ and $f: I \rightarrow D$ an $R$-homomorphism. Let $I_{\alpha}$ be a finitely generated ideal contained in $I$, and let $D_{\alpha}$ be the annihilator in $D$ of $I_{\alpha}$. Then by [1, prop. 7.3.4] there exists an element $x_{\alpha}$ in $D$ such that $f(a)=a x_{\alpha}$ for every $a$ in $I_{\alpha}$. The set of congruences :

$$
x \equiv x_{\alpha} \quad\left(\bmod D_{\alpha}\right)
$$


(ranging over all of the annihilators of finitely generated ideals contained in $I$ ) is finitely solvable. Hence there exists a simultaneous solution $y$ in $D$. Then $f(a)=a y$ for every a in $I$. Thus $D$ is injective.

The proof of the 'if' part of the following theorem was communicated to me by I. Kaplansky.

THEOREM 4. Let $R$ be a valuation ring with quotient field $Q$. Then $R$ is almost maximal, if and only if $Q / R$ is an injective $R$-module. In this case $Q / I$ is an indecomposable, injective $R$-module for every ideal $I$ of $R$; and if $I \neq R$, then $Q / I \cong E(R / I)$.

Proof. If $R$ is an almost maximal valuation ring, then the assertions follow from Proposition 3 and Proposition 1.

Conversely, assume that $Q / R$ is an injective $R$-module. Suppose that the following set of congruences is finitely solvable:

$$
x \equiv r_{\alpha} \quad\left(\bmod I_{\alpha}\right),
$$

where $r_{\alpha} \in R, I_{\alpha}$ is an ideal of $R$, and $I=\cap I_{\alpha} \neq 0$. If $I_{\beta}=I$ for some $\beta$, then $r_{\beta}$ is a solution of the congruences. Hence we can assume that $I_{\alpha} \neq I$ for all $\alpha$.

Take $c \neq 0$ in $I$, and define $J=\{b \in R \mid b I \varsubsetneqq c R\} . J$ is an ideal of $R$, and for every $b \in J$ there exists an index $\alpha_{b}$ such that $b I_{\alpha_{b}} \subset c R$. We define an $R$ homomorphism $f: J \rightarrow Q / c R$ by $f(b)=b r_{\alpha_{b}}+c R$ for every $b \in J$. Since $Q / c R$ is injective, there is an element $q \in Q$ such that $f(b)=b q+c R$ for every $b \in J$. We will show that $g$ is a solution of the congruences.

Take any index $\alpha$, and take $d \in I_{\alpha}-I$. Then $c=d y$ for some $y \in R$. In fact, $y \in J$. For if $y \notin J$, then $y I \supset c R, c=y z$ for some $z \in I$, and then $d=z \in I$; which is a contradiction. Now there exists an index $\beta$ such that $I_{\beta} \subset I_{\alpha}$, and such that $y r_{\beta}+c R=f(y)=y q+c R$. Thus $y\left(r_{\beta}-q\right)=r c=r y d$ for some $r \in R$, and so $r_{\beta}-q \in I_{\alpha}$. Therefore, $r_{\alpha}-q=\left(r_{\alpha}-r_{\beta}\right)+\left(r_{\beta}-q\right) \in I_{\alpha}$. Hence $q$ is an element of $R$, and is a solution of the congruences. This proves that $R$ is linearly precompact, and thus is almost maximal.

As a consequence of Theorem 4 and Proposition 1 we see that if $D$ is a homomorphic image of an injective module over an almost maximal valuation ring, then every element of $D$ is contained in an indecomposable, injective direct summand of $D$. 
Theorem 5. Let $R$ be an integral domain with quotient field $Q$. Then every $R$ :homomorphic image of $Q$ is injective, if and only if $R$ is a Prufer ring and every $R$-homomorphic image of $Q$ is semi-compact. In this case $R_{M}$ is an almost maximal valuation ring for every maximal ideal $M$ of $R$.

Proof. If $R$ is a Prufer ring and every image of $Q$ is semi-compact, then every image of $Q$ is injective by Proposition 3. Conversely, suppose that every image of $Q$ is injective. Then each of these images is semi-compact by Proposition 2. We will prove that $R$ is a Prufer ring.

Let $A$ be any $R$-module, and $I$ an ideal of $R$. Suppose that $\operatorname{Tor}_{2}^{R}(A, R / I)$ $\neq 0$. Then there exists a maximal ideal $M$ of $R$ such that $\operatorname{Hom}_{R}\left(\operatorname{Tor}_{2}^{R}(A, R / I)\right.$, $Q / M) \neq 0$. Now $\operatorname{Hom}_{R}(R / I, Q / M) \cong S / M$, where $S$ is an $R$-submodule of $Q$. From the exact sequence:

$$
0 \rightarrow S / M \rightarrow Q / M \rightarrow Q / S \rightarrow 0
$$

we see that $S / M$ has injective dimension one, or less. Therefore, by [1, Prop. 6.5.1], $0=\operatorname{Ext}_{R}^{2}(A, S / M) \cong \operatorname{Ext}_{R}^{2}\left(A, \operatorname{Hom}_{R}(R / I, Q / M)\right) \cong \operatorname{Hom}_{R}\left(\operatorname{Tor}_{2}^{R}(A, R / I)\right.$, $Q / M)$. This contradiction shows that $\operatorname{Tor}_{2}^{R}(A, R / I)=0$. Hence w. gl. $\operatorname{dim} . R \leqq 1$. By [3, Thm. 2] $R$ is a Prufer ring.

If $M$ is a maximal ideal of $R$, then w.gl.dim. $R_{M} \leqq$ w.gl.dim. $R \leqq 1$; and so $R_{M}$ is a local, Prufer ring. Therefore $R_{M}$ is a valuation ring. From [1, Prop. 6.4.1.3] we see that $Q / R_{i 1}$ is an injective $R_{M}$-module. Thus $R_{M}$ is an almost maximal valuation ring by Theorem 4 .

Remarks. 1) It can be shown that if $R$ is a Noetherian integral domain, then $R$ is a Dedekind ring if and only if $Q / I$ is injective for every ideal $I$ of $R$. However, it is not sufficient merely to assume, even for Noetherian local domains, that $Q / R$ is injective as the following example due to I. Kaplansky shows. Let $R$ be the ring of formal power series in one variable over a field with the first degree term missing. Then $Q / R$ is injective, but $R$ is not a Dedekind ring. $I$ am indebted to A. Rosenberg and D. Zelinsky for the remark that if $R$ is Noetherian, $\operatorname{gl} \operatorname{dim} . R<\infty$, and $Q / R$ is injective, then it follows easily from [1, Ch. 6, Ex. 9] that $R$ is a Dedekind ring.

2) The following questions remain unanswered. If $R_{M}$ is an almost maximal valuation ring for every maximal ideal $M$ of $R$, is it true that every image of the quotient field of $R$ is injective? For a Prufer ring is the linear precompact- 
ness of it quotient field $Q$ equivalent to the semi-compactness of every image of $Q$ ? Is a Prufer ring with linearly precompact quotient field either an almost maximal valuation ring or a Dedekind ring? For similar questions concerning maximal valuation rings we shall be able to provide the answers.

Lemma 6. Let $R$ be an integral domain with quotient field $Q \neq R$, and $K=Q / R$. Then $\operatorname{Hom}_{R}(K, K) \cong R$, if and only if $\operatorname{Ext}_{R}^{1}(Q, R)=0$. In this case $\operatorname{Ext}_{R}^{1}(Q, I)=0$ for every ideal $I$ of $R$.

Proof. From the exact sequence:

$$
0 \rightarrow R \rightarrow Q \rightarrow K \rightarrow 0
$$

we readily obtain first that $\operatorname{Hom}_{R}(K, K) \cong \operatorname{Ext}_{R}(K, R)$, and then the exact sequence :

$$
0 \rightarrow R \rightarrow \operatorname{Hom}_{R}(K, K) \rightarrow \operatorname{Ext}_{R}^{1}(Q, R) \rightarrow 0 .
$$

Thus if $\operatorname{Ext}_{R}^{1}(Q, R)=0$, then $\operatorname{Hom}_{R}(K, K) \cong R$; whereas if $\operatorname{Hom}_{R}(K, K) \cong R$, then $\operatorname{Ext}_{R}^{1}(Q, R)$ is cyclic, divisible, and torsion-free, and hence is zero. The remaining assertion follows easily.

Lемма 7. Let $R$ be an almost maximal valuation ring with quotient field $Q$ and maximal ideal $M$. Let $K=Q / R$ and $E=Q / M$ (i.e., $E=E(R / M)$ ). Then $\operatorname{Hom}_{R}(K, K) \cong R$, if and only if $\operatorname{Hom}_{R}(E, E) \cong R$.

Proof. Assume that $\operatorname{Hom}_{R}(E, E) \cong R$. Then using [1, Prop. 7.2.3; Prop. 6.5.1; Prop. 7.2.2] we obtain: $\operatorname{Hom}_{R}(K, K) \cong \operatorname{Ext}_{R}^{1}(K, R) \cong \operatorname{Ext}_{R}^{1}(K$, $\left.\operatorname{Hom}_{R}(E, E)\right) \cong \operatorname{Hom}_{R}\left(\operatorname{Tor}_{1}^{R}(K, E), E\right) \cong \operatorname{Hom}_{R}(E, E) \cong R$.

Conversely, assume that $\operatorname{Hom}_{R}(K, K) \cong R$. If $M$ is principal, the result is trivial; hence assume that $M$ is not principal. Then $\operatorname{Hom}_{R}(R / M, K)=0$. Therefore, from the exact sequence:

$$
0 \longrightarrow R / M \longrightarrow E \stackrel{P}{\rightarrow} K \longrightarrow 0,
$$

where $P$ is the canonical map, we obtain the exact sequence:

$$
0 \longrightarrow \operatorname{Hom}_{R}(K, K) \stackrel{P^{*}}{\longrightarrow} \operatorname{Hom}_{R}(E, K) \longrightarrow \operatorname{Hom}_{R}(R / M, K)=0 .
$$

There is a natural monomorphism of $R$ into $\operatorname{Hom}_{R}(E, E)$ sending an element of $R$ into multiplication by that element. We will prove that the mapping is onto. Let $f \in \operatorname{Hom}_{R}(E, E)$. Then $P f \in \operatorname{Hom}_{R}(E, K)$, and thus 
from the above exact sequence $P f=g P$, where $g \in \operatorname{Hom}_{R}(K, K)$. Now $g$ is multiplication in $K$ by an element $r$ of $R$; hence $P f=g P=P h$, where $h$ is multiplication by $r$ in $E$. Thus $\operatorname{Im}(f-h) \subset \operatorname{Ker} P=R / M$. Therefore, since $E$ is divisible, $f-h=0$, and $\operatorname{Hom}_{R}(E, E) \cong R$.

THEOREM 8. (Duality) Let $R$ be an almost maximal valuation ring with quotient field $Q$ and maximal ideal $M$. Let $K=Q / R, E=E(R / M)=Q / M$, and assume that $\operatorname{Hom}_{R}(K, K) \cong R$. If $A$ (or I) is a submodule of $E$ (or R), let $A^{*}$ (or $\left.I^{*}\right)$ be the annihilator of $A$ (or $\left.I\right)$ in $R$ (or $E$ ). Then:

1) $A^{* *}=A$; and $I^{* *}=I$.

2) $\operatorname{Hom}_{R}(E / A, E) \cong A^{*}$; and $\operatorname{Hom}_{R}(R / I, E) \cong I^{*}$.

3) $\operatorname{Hom}_{R}(A, E) \cong R / A^{*}$; and $\operatorname{Hom}_{R}(I, E) \cong E / I^{*}$.

Thus $\operatorname{Hom}_{R}\left(\operatorname{Hom}_{R}(B, E), E\right) \cong B$ whenever $B$ is a submodule or factor module of either $R$ or $E$.

Proof. By Lemma $7 \operatorname{Hom}_{R}(E, E) \cong R$. Then the proof is essentially the same as [6, Thm. 4.2]. For a more general result see [7, Thm. 5.2].

Theorem 9 will show that Theorem 8 is a duality assertion for maximal valution rings. For a discussion of duality for complete, discrete valuation rings see [5].

Theorem 9. Let $R$ be an integral domain with quotient field $Q \neq R$ and $K=Q / R$. Then the following statements are equivalent:

1) $R$ is a maximal valuation ring.

2) $R$ is a Prufer ring with linearly compact quotient field.

3) All $R$-homomorphic images of $Q$ are injective; and $\operatorname{Hom}_{R}(K, K) \cong R$.

4) $R$ is an almost maximal valuation ring; and $\operatorname{Hom}_{R}(K, K) \cong R$.

5) $\operatorname{Ext}_{R}^{1}(A, S)=0$ for $A$ any torsion-free module and $S$ any torsion-free module of rank one.

Proof. 1) $\Rightarrow 2$ ). Trivial.

$2) \Rightarrow 3$ ). Every image of $Q$ is injective by Proposition 3. There is a natural monomorphism of $R$ into $\operatorname{Hom}_{R}(K, K)$ sending an element of $R$ into multiplication by that element. We will prove that this mapping is onto. Let $f \in \operatorname{Hom}_{R}(K, K)$, and $a \neq 0 \in R$. Let $f(1 / a+R)=q+R$, where $q \in Q$. Then $a q=r_{a} \in R$, and $f$ agrees with multiplication by $r_{a}$ on the submodule of $K$ 
generated by $1 / a+R$. Thus the set of congruences:

$$
x \equiv r_{a} \quad(\bmod a R)
$$

is finitely solvable in $R$. Since $R$ is linearly compact, there exists a simultaneous solution $r$ in $R$. Thus $f$ is multiplication by $r$ and $\operatorname{Hom}_{R}(K, K) \cong R$.

$3) \Rightarrow 4)$. It is sufficient to prove that $R$ is a valuation ring, for then 4 ) will follow from Theorem 4. Let $I$ be any ideal of $R$. Suppose that $Q / I$ is not indecomposable. Then there exists a submodule $S$ of $Q, S \neq I, Q$, such that $S / I$ is an injective module. From the exact sequence:

$$
0 \rightarrow I \rightarrow S \rightarrow S / I \rightarrow 0
$$

we derive the exact sequence:

$$
0=\operatorname{Hom}_{R}(Q, S) \rightarrow \operatorname{Hom}_{R}(Q, S / I) \rightarrow \operatorname{Ext}_{R}^{1}(Q, I) .
$$

By Lemma $6 \operatorname{Ext}_{R}^{1}(Q, I)=0$. Therefore, $\operatorname{Hom}_{R}(Q, S / I)=0$; which is a contradiction, since $S / I$ is a non-zero injective module. Thus $Q / I$ is an indecomposable, injective module. By [6, Prop. 2.2] any two non-zero submodules of $Q / I$ have a non-zero intersection. It follows that $I$ is not the intersection of two properly larger ideals. Thus the ideals of $R$ are simply ordered, and $R$ is a valuation ring.

$4) \Longrightarrow 5$ ). If $S=Q$, then $\operatorname{Ext}_{R}^{1}(A, S)=0$. If $S \neq Q$, then since $R$ is a valuation ring, we can assume that $S \subset R$. From the exact sequence:

$$
0 \rightarrow A \rightarrow A \otimes Q \rightarrow A \otimes K \rightarrow 0
$$

we obtain the exact sequence:

$$
\operatorname{Ext}_{R}^{1}(A \otimes Q, S) \rightarrow \operatorname{Ext}_{R}^{1}(A, S) \rightarrow \operatorname{Ext}_{R}^{2}(A \otimes K, S) .
$$

But $\operatorname{Ext}_{R}^{1}(A \otimes Q, S)=0$ by Lemma 6 ; and $\operatorname{Ext}_{R}^{2}(A \otimes K, S)=0$, since $S$ has injective dimension one by Theorem 4. Therefore, $\operatorname{Ext}_{R}^{1}(A, S)=0$.

$5) \Rightarrow 3$ ). Let $B$ be any $R$-module and $S$ an $R$-submodule of $Q$. Consider an exact sequence :

$$
0 \rightarrow A \rightarrow F \rightarrow B \rightarrow 0,
$$

where $F$ is free. From this we obtain the exact sequence:

$$
0=\operatorname{Ext}_{R}^{1}(A, S) \rightarrow \operatorname{Ext}_{R}^{2}(B, S) \rightarrow \operatorname{Ext}_{R}^{2}(F, S)=0 .
$$

Therefore, $\operatorname{Ext}_{R}^{2}(B, S)=0, S$ is of injective dimension one or less, and $Q / S$ is 
injective. Since $\operatorname{Ext}_{R}^{1}(Q, R)=0$ by assumption, $\operatorname{Hom}_{R}(K, K) \cong R$ by Lemma 6 . $4) \Rightarrow 1$ ). We must prove that $R$ is linearly compact. Let

$$
x \equiv r_{\alpha} \quad\left(\bmod I_{\alpha}\right)
$$

be a finitely solvable set of congruences, where $r_{\alpha} \in R$ and the $I_{\alpha}$ 's are ideals of $R$. Since $R$ is already linearly precompact, we can assume that $\cap I_{\alpha}=0$. Now $Q / M=E(R / M)=E$, and $\operatorname{Hom}_{R}(E, E) \cong R$ by Lemma 7. Let $B_{\alpha}$ be the annihilator of $I_{\alpha}$ in $E$. Then $\operatorname{Hom}_{R}\left(B_{\alpha}, E\right) \cong R / I_{\alpha}$ and $\cup B_{\alpha}=E$ by Theorem 8. Hence, $R \cong \operatorname{Hom}_{R}(E, E) \cong \operatorname{Lim} \operatorname{Hom}_{R}\left(B_{a}, E\right) \cong \operatorname{Lim} R / I_{\alpha}$. The finite solvability of the congruences says that $\left(r_{\alpha}+I_{\alpha}\right)_{\alpha} \in \operatorname{Lim} R / I_{\alpha}$. Hence there exists $r \in R$ such that $\left(r+I_{\alpha}\right)_{\alpha}=\left(r_{\alpha}+I_{\alpha}\right)_{\alpha}$. Thus $r$ is a solution of the congruences. Therefore, $R$ is linearly compact, and so $R$ is a maximal valuation ring. This concludes the proof of the theorem.

We note that Theorems 13 and 14 of [4] and Propositions 3 and 4 of [2] now follow immediately from Theorem $9:(5)$ and an easy induction.

Proposition 10. Let $R$ be a ring (not necessarily commutative), and $\left\{A_{i}\right\}$ a countable family of $R$-nodules such that every homomorphic image of each $A_{i}$ is injective. Let $B$ be a homomorphic image of $\Sigma \oplus A_{i}$. Then $B=\Sigma \oplus B_{i}$, where $B_{i}$ is a homomorphic image of $A_{i}$, and thus is an injective submodule of $B$. Therefore, if the family is finite, every homomorphic image of $\Sigma \oplus A_{i}$ is injective.

Proof. Let $f: \Sigma \oplus A_{i} \rightarrow B$ be an $R$-homomorphism onto $B$. Let $C_{i}=f\left(A_{i}\right)$, and $D_{n}=C_{1}+\cdots+C_{n}$. We will prove by induction on $n$ that $D_{n}=B_{1} \oplus \ldots$ $\oplus B_{n}$, where $B_{i}$ is an image of $A_{i}$, and is therefore injective. Let $B_{1}=C_{1}$, and assume that the assertion is true for $n-1 \geqslant 1$. Thus $D_{n-1}=B_{1} \oplus \ldots \oplus B_{n-1}$. Since $D_{n-1}$ is injective, $D_{n}=D_{n-1} \oplus B_{n}$, where $B_{n}$ is a submodule of $D_{n}$. Thus $B_{n} \cong D_{n} / D_{n-1}=\left(D_{n-1}+C_{n}\right) / D_{n-1} \cong C_{n} /\left(D_{n-1} \cap C_{n}\right)$; and so $B_{n}$ is a homomorphic image of $A_{n}$ and the induction is verified. Since the $D_{n}$ 's are an increasing sequence of submodules of $B$ and $B=\cup D_{n}$, it follows that $B=\Sigma \oplus B_{i}$.

Let $R$ be an integral domain with quotient field $Q$ such that every image of $Q$ is injective. Let $Q^{n}$ denote a direct sum of $n$ copies of $Q$, and $Q^{\infty}$ a direct sum of denumerably infinite number of copies of $Q$. Then by Proposition 10 every image of $Q^{n}$ (or $Q^{\infty}$ ) is a direct sum of a finite (or a countable) number of injective modules; and thus every image of $Q^{n}$ is injective. It follows that 
every torsion free module of finite rank has injective dimension $\leqq 1$. Therefore, every image of a torsion-free module of finite rank has injective dimension $\leqq 1$ also. This generalizes [2, Lemma 4]. In general images of $Q^{\infty}$ are not injective, even though they are direct sums of injective modules, as will be seen from the following proposition. But first we have a definition.

Definition. Let $R$ be a valuation ring and $I$ an ideal of $R$. We say that $I$ is Archimedean if $a I \neq I$ for every non-unit $a \in R$. Clearly, every principal ideal of $R$ is Archimedean. The terminology stems from the fact that it can be shown that if every non-zero ideal of $R$ is Archimedean, then the value group associated with $R$ is Archimedean.

Proposition 11. Let $R$ be a non-discrete valuation ring. Let $\left\{I_{n}\right\}$ be $a$ countable family of Archimedean ideals, $E_{n}=E\left(R / I_{n}\right)$ and $C=E_{1} \oplus E_{2} \oplus \ldots$ Then $C$ is injective, if and only if the family is finite.

Proof. Assume that the family is infinite. Since $R$ is not Noetherian, there exists an ideal $I$ of $R$ which is generated by an infinite set of elements $\left\{r_{1}, r_{2}, \ldots\right\}$ such that $r_{i+1}$ properly divides $r_{i}$; i.e. there exists non-units $a_{i+1} \in R$ such that $r_{i}=a_{i+1} r_{i+1}$ for every $i$.

We define a mapping $f$ from the generators of $I$ to $C$ by induction. Let $f\left(r_{1}\right)=x_{11}$ be any non-zero element of $E_{1}$. Assume that we have defined $f\left(r_{i}\right)$ for $i=1,2, \ldots, n$ such that:

1) $f\left(r_{i}\right)=x_{1 i}+\cdots+x_{i i}$, where $x_{j i} \neq 0 \in E_{j}$.

2) $a_{i} x_{j i}=x_{j, i-1}$ for $j=1, \ldots, i-1$ and $i=2, \ldots, n$.

3) $a_{i} x_{i i}=0$ for $i=2, \ldots, n$.

Since $E_{j}$ is divisible, we can find $x_{j, n+1} \in E_{j}$ such that $x_{j n}=a_{n+1} x_{j, n+1}$ for $j=1$, $\ldots, n$. Since $I_{n+1}$ is Archimedean, we can find an element $q \in\left(1 / a_{n+1}\right) I_{n+1}$ and $q \notin I_{n+1}$. Let $x_{n+1, n+1}=q+I_{n+1} \in Q / I_{n+1} \subset E_{n+1}$, and define $f\left(r_{n+1}\right)=x_{1, n+1}+$ $\cdots+x_{n+1, n+1}$. Then $f\left(r_{n+1}\right)$ satisfies the above three conditions. It is easily verified that $f$ induces an $R$-homomorphism $f$ from $I$ to $C$. By construction the image of $I$ is not contained in any finite number of the $E_{i}$ 's. Thus $f$ can not be extended to all of $R$, and so $C$ is not injective.

As a consequence of Proposition 11 we see that if $R$ is an almost maximal, non-discrete, valuation ring with quotient field $Q$ and $K=Q / R$, then a direct 
sum of copies of $K$ is injective if and only if the number of summands is finite.

Lemma 12. Let $R$ be an integral domain with quotient field $Q$. Let $B$ be an $R$-module with torsion submodule $T$ of bounded order (i.e. $r T=0$ for some $r \neq 0 \in R$ ) and such that $B / T$ is divisible. Then $T$ is a direct summand of $B$. Thus $\operatorname{Ext}_{R}^{1}(Q, T)=0$.

Proof. It is clear that $T \cap r B=0$. Let $x \in B$; then there exists $y \in B$ such that $x+T=r(y+T)$. Hence $x \in T \oplus r B$, and so $B=T \oplus r B$.

Parts 1) and 2) of the following proposition are known for almost maximal valuation rings and Dedekind rings [4].

Proposition 13. Let $R$ be an integral domain with quotient field $Q$ such that every homomorphic image of $Q$ is injective. Let $B$ be a module over $R$ with torsion submodule T. Then:

1) If $T$ is finitely generated, it is a direct summand.

2) If $T$ is of bounded order and $R$ is a Dedekind ring, $T$ is a direct summand.

3) If $T$ is a homomorphic image of a torsion-free module of finite rank, and if $R$ is an almost maximal valuation ring, then $T$ is a direct summand.

Proof. Let $B / T=S$; then from the exact sequence:

$$
0 \rightarrow \mathrm{S} \rightarrow Q \otimes S \rightarrow Q / R \otimes S \rightarrow 0
$$

we derive the exact sequence:

$$
\operatorname{Ext}_{R}^{1}(Q \otimes S, T) \rightarrow \operatorname{Ext}_{R}^{1}(S, T) \rightarrow \operatorname{Ext}_{R}^{2}(Q / R \otimes S, T) .
$$

In the first two cases $T$ is of bounded order; while in the third case, we can assume without loss of generality that $T$ is of bounded order also. Hence, $\operatorname{Ext}_{R}^{1}(Q \otimes S, T)=0$ by Lemma 12 . For cases 1) and 3) $T$ has injective dimension $\leqq 1$ by the remarks following Proposition 10 . Thus in all cases $\operatorname{Ext}_{R}^{2}(Q / R \otimes S, T)=0$. Therefore, $\operatorname{Ext}_{R}^{1}(S, T)=0$; which proves the proposition.

Proposition 14. Let $R$ be a valuation ring with quotient field $Q$; let $A_{1}$, $\ldots, A_{n}$ be (not necessarily proper) homomorphic images of $Q$; and let $A=A_{1} \oplus \cdots \oplus A_{n}$. Then if $B$ is any finitely generated submodule of $A, B$ is a direct sum of cyclic modules. 
Proof. The proof will be by induction on $n$. If $n=1$, then $B$ is a cyclic module. Assume the proposition is true for $n-1 \geq 1$. Let $x_{1}, \ldots, x_{m}$ be the generators of $B$, where $x_{j}=\left(x_{j}^{(k)}\right), x_{j}^{(k)} \in A_{k}$. Without loss of generality we can assume that the order ideal of $x_{1}$ is equal to the order ideal of $x_{1}^{(1)}$, and that there exist elements $r_{j} \in R$ such that $r_{j} x_{1}^{(1)}=x_{j}^{(1)}$ for $j=2, \ldots, m$. Let $y_{j}=r_{j} x_{1}-x_{j}$, and $C=\left\{y_{2}, \ldots, y_{m}\right\}$. Then $C \subset A_{2} \oplus \cdots \oplus A_{n}$, and hence, by induction, $C$ is a direct sum of cyclic modules. Clearly $B=R x_{1}+C$. If $a x_{1}+z$. $=0$ for $a \in R$ and $z \in C$, then $a x_{1}^{(1)}=0$, and so $a x_{1}=0$. Thus $B=R x_{1} \oplus C$ is a direct sum of cyclic modules.

We note that Theorem 14 [4] (i.e. any finitely generated module over an almost maximal valuation ring is a direct sum of cyclic modules) follows directly from Proposition 14 and Theorem 4. For Noetherian integral domains this direct sum property for finitely generated torsion modules characterizes Dedekind rings.

Proposition 15. Let $R$ be a Noetherian integral domain. Then every finitely generated torsion $R$-modula is a direct sum of cyclic modules, if and only if $R$ is a Dedekind ring.

Proof. If $R$ is a Dedekind ring, the result is known [4]. For the converse let $M$ be any maximal ideal of $R, E=E(R / M)$, and $A_{i}$ be the annihilator in $E$ of $M^{i}$. Now $A_{i}$ is a finitely generated torsion module [6, Thm. 3.11]; and since $E$ is an indecomposable, injective $R$-module, $A_{i}$ must be a cyclic module. Therefore, $M / M^{2}$ is one dimensional over $R / M$ [6, Thm. 3.10], and hence $R_{M}$ is a discrete valuation ring. Since $\mathrm{gl} . \operatorname{dim} . R=\sup _{M} \mathrm{gl}$. $\operatorname{dim} . R_{M}=1$, we see that $R$ is hereditary; and, therefore, $R$ is a Dedekind ring.

\section{BIBLIOGRAPHY}

[1] H. Cartan and S. Eilenberg, Homological Algebra, Princeton University Press, 1956.

[2] I. Fleischer, Modules of finite rank over Prufer rings, Annals of Mathematics, vol. 65, No. 2 (1957), pp. 250-254.

[3] A. Hattori, On Prufer rings, Journal of the Mathematical Society of Japan, vol. 9. No. 4 (1957), pp. 381-385.

[4] I. Kaplansky, Modules over Dedekind rings and valuation rings, Transactions of the American Mathematical Society, vol. 72, No. 2 (1952), pp. 227-340.

[5] I. Kaplansky, Dual modules over a valuation ring $I$, Proceedings of the American Mathematical Society, vol. 4, No. 2 (1953), pp. 213-217.

[6] E. Matlis, Injective modules over Noetherian rings, Pacific Journal of Mathematics, vol. 8 , No. 3 (1958), pp. 511-528. 
[7] K. Morita, Duality for modules and its applications to the theory of rings with minimum condition, Science Reports of the Tokyo Kyoiku Daigaku, Sect. A, vol. 6, No. 150 (1958), pp. 83-142.

[8] D. Zelinsky, Linearly compact modules and rings, American Journal of Mathematics, yol. 75, No. 1 (1953), pp. 79-90. 
